\title{
FROM NORM DERIVATIVES TO ORTHOGONALITIES IN HILBERT $C^{*}$-MODULES
}

\author{
PAWEŁ WÓJCIK ${ }^{1}$ and ALI ZAMANI ${ }^{2, *}$
}

\begin{abstract}
Let $(\mathscr{X},\langle\cdot, \cdot\rangle)$ be a Hilbert $C^{*}$-module over a $C^{*}$-algebra $\mathscr{A}$ and let $\mathcal{S}(\mathscr{A})$ be the set of states on $\mathscr{A}$. In this paper, we first compute the norm derivative for elements $x$ and $y$ of $\mathscr{X}$ as follows

$$
\rho_{+}(x, y)=\max \left\{\operatorname{Re} \varphi(\langle x, y\rangle): \varphi \in \mathcal{S}(\mathscr{A}), \varphi(\langle x, x\rangle)=\|x\|^{2}\right\} .
$$

We then apply it to characterize different concepts of orthogonality in $\mathscr{X}$. In particular, we present a simpler proof of the classical characterization of Birkhoff-James orthogonality in Hilbert $C^{*}$-modules. Moreover, some generalized Daugavet equation in the $C^{*}$-algebra $\mathbb{B}(\mathcal{H})$ of all bounded linear operators acting on a Hilbert space $\mathcal{H}$ is solved.
\end{abstract}

\section{Introduction and Preliminaries}

Let $(X,\|\cdot\|)$ be a normed space and $X^{*}$ its dual topologic space. We define two mappings $\rho_{+}, \rho_{-}: X \times X \rightarrow \mathbb{R}$ by the formulas

$$
\rho_{ \pm}(x, y):=\lim _{t \rightarrow 0^{ \pm}} \frac{\|x+t y\|^{2}-\|x\|^{2}}{2 t}=\|x\| \cdot \lim _{t \rightarrow 0^{ \pm}} \frac{\|x+t y\|-\|x\|}{t} .
$$

The convexity of the norm yields that the above definitions are meaningful. These mappings are called the norm derivatives and their following useful properties can be found, e.g. in $[1,12]$. For every $x$ and $y$ in $X$ and for every $\alpha=|\alpha| e^{i \theta}$, $\beta=|\beta| e^{i \omega}$ in $\mathbb{C}$, we have

(P1) $\rho_{-}(x, y) \leq \rho_{+}(x, y),\left|\rho_{ \pm}(x, y)\right| \leq\|x\| \cdot\|y\|$ and $\rho_{ \pm}(x, x)=\|x\|^{2}$,

(P2) $\rho_{ \pm}(-x, y)=\rho_{ \pm}(x,-y)=-\rho_{\mp}(x, y)$,

(P3) $\rho_{ \pm}(x, \alpha x+y)=\operatorname{Re} \alpha\|x\|^{2}+\rho_{ \pm}(x, y)$,

(P4) $\rho_{ \pm}(\alpha x, \beta y)=|\alpha \beta| \rho_{ \pm}\left(x, e^{i(\omega-\theta)} y\right)$,

(P5) $\rho_{+}(x, y)=\lim _{t \rightarrow 0^{+}} \rho_{+}(x+t y, y)$.

If the norm on $X$ comes from an inner product $[\cdot, \cdot]$, then we obtain $\rho_{+}(x, y)=$ $\rho_{-}(x, y)=\operatorname{Re}[x, y]$ for all $x, y \in X$, i.e. both $\rho_{+}$and $\rho_{-}$are perfect generalizations of inner products.

In more general cases mappings $\rho_{+}$and $\rho_{-}$are useful for applications in approximation theory and, in particular, they played a significant role in the paper [28]. For more information about the norm derivatives and their properties the reader is referred to $[1,12]$ (see also $[8,9,19,32]$ ).

2010 Mathematics Subject Classification. 46L08; 46C50; 46B20; 46L05.

Key words and phrases. Hilbert $C^{*}$-module; $C^{*}$-algebra; state; norm derivative; BirkhoffJames orthogonality. 
From [12], for two elements $x$ and $y$ of a normed linear space $X$, we have

$$
\rho_{+}(x, y)=\|x\| \max \left\{\operatorname{Re} x^{*}(y): x^{*} \in J(x)\right\},
$$

where $J(x):=\left\{x^{*} \in X^{*}:\left\|x^{*}\right\|=1, x^{*}(x)=\|x\|\right\}$. If we have additional structures on a normed linear space $X$, then we obtain other expressions for the norm derivative $\rho_{+}$. Therefore we survey on the well known results involving norm derivatives. So the present section has an expository character in part; however, many of the surveyed results will be essentially extended in the next section.

In the classical Banach space $\mathcal{C}(K)$ of all continuous functions on a compact Hausdroff space $K$, the result for $f, g \in \mathcal{C}(K)$ were given by Keckić [16]:

$$
\rho_{+}(f, g)=\|f\| \max \left\{\operatorname{Re}\left(e^{-i \arg (f(x))} g(x)\right): x \in M_{f}\right\}
$$

where $M_{f}:=\{x \in K:|f(x)|=\|f\|\}$.

In $\mathbb{B}(\mathcal{H})$, the $C^{*}$-algebra of all bounded linear operators on a Hilbert space $(\mathcal{H},[\cdot, \cdot])$, and for $T, S \in \mathbb{B}(\mathcal{H})$, Keckić $[15]$ obtained the following formula:

$$
\rho_{+}(T, S)=\inf _{\varepsilon>0} \sup \left\{\operatorname{Re}[S x, T x]: x \in \mathcal{H}_{\varepsilon},\|x\|=1\right\},
$$

where $\mathcal{H}_{\varepsilon}:=E_{T^{*} T}\left((\|T\|-\varepsilon)^{2},\|T\|^{2}\right)$, and $E_{T^{*} T}$ stands for the spectral measure of the operator $T^{*} T$.

Norm derivatives of the space $\mathbb{K}(\mathcal{H})$ (compact operators on $\mathcal{H}$ ) has been studied in [14]. More precisely, for $T, S \in \mathbb{K}(\mathcal{H})$ where $T=U|T|$ is the polar decomposition of $T$, we have

$$
\rho_{+}(T, S)=\|T\| \max \left\{\operatorname{Re}\left[U^{*} S x, x\right] ; x \in \Phi,\|x\|=1\right\},
$$

where $\Phi$ is the characteristic subspace of $T$ with respect to its eigenvalue $s_{1}$.

Wójcik [24], by using a different method, extended this result for compact operators between real normed spaces, i.e. $\mathbb{K}(X, Y)$. Moreover, similar investigations have been carried out by Wójcik [26] in $M$-ideals in bounded operator space $\mathbb{B}(X, Y)$. The main result of [26] says that if $T, S \in \mathbb{B}(X, Y)$ and $\operatorname{dist}(T, \mathbb{K}(X, Y))<\|T\|$, then

$$
\rho_{+}(T, S)=\|T\| \max \left\{\rho_{+}(T x, S x): x \in \operatorname{Ext} B_{X},\|T x\|=\|T\|\right\},
$$

where $\operatorname{Ext} B_{X}$ denotes the set of all extremal points of the closed unit ball $B_{X}$.

Motivated by the above properties, we compute the norm derivatives in Hilbert $C^{*}$-modules. Namely, for two elements $x$ and $y$ of a Hilbert $\mathscr{A}$-module $(\mathscr{X},\langle\cdot, \cdot\rangle)$ we will prove that

$$
\rho_{+}(x, y)=\max \left\{\operatorname{Re} \varphi(\langle x, y\rangle): \varphi \in \mathcal{S}(\mathscr{A}), \varphi(\langle x, x\rangle)=\|x\|^{2}\right\},
$$

where $\mathcal{S}(\mathscr{A})$ is the set of states on $\mathscr{A}$. This formula enables us to characterize different concepts of Birkhoff-James orthogonality for elements of a Hilbert $C^{*}$ module. Some other related results are also discussed.

Before stating the results, we establish the notation and recall some definitions from the literature. An element $a$ in a $C^{*}$-algebra $\mathscr{A}$ is called positive (we write 
$a \geq 0)$ if $a=b^{*} b$ for some $b \in \mathscr{A}$. A linear functional $\varphi$ of $\mathscr{A}$ is positive if $\varphi(a) \geq 0$ for every positive element $a \in \mathscr{A}$. A state is a positive linear functional whose norm is equal to one. The symbol $\mathcal{S}(\mathscr{A})$ denotes the set of states on $\mathscr{A}$.

An inner product module over $\mathscr{A}$ is a (left) $\mathscr{A}$-module $\mathscr{X}$ equipped with an $\mathscr{A}$-valued inner product $\langle\cdot, \cdot\rangle$, which is $\mathbb{C}$-linear and $\mathscr{A}$-linear in the first variable and has the properties $\langle x, y\rangle^{*}=\langle y, x\rangle$ as well as $\langle x, x\rangle \geq 0$ with equality if and only if $x=0$. An inner product $\mathscr{A}$-module $\mathscr{X}$ is called a Hilbert $\mathscr{A}$-module if it is complete with respect to the norm $\|x\|=\|\langle x, x\rangle\|^{\frac{1}{2}}$. For $x \in \mathscr{X}$, by [20, Theorem 3.3.6], there always exists a $\varphi \in \mathcal{S}(\mathscr{A})$ such that $\varphi(\langle x, x\rangle)=\|x\|^{2}$. So, let $\Omega_{x}$ denote the (nonempty) subset of the set of supporting functionals:

$$
\Omega_{x}:=\left\{\varphi \in \mathcal{S}(\mathscr{A}): \varphi(\langle x, x\rangle)=\|x\|^{2}\right\} \subseteq J(x) .
$$

Given a positive functional $\varphi$ on $\mathscr{A}$, we have the following useful version of the Cauchy-Schwarz inequality:

$$
|\varphi(\langle x, y\rangle)|^{2} \leq \varphi(\langle x, x\rangle) \varphi(\langle y, y\rangle) \quad(x, y \in \mathscr{X}) .
$$

Every $C^{*}$-algebra $\mathscr{A}$ can be regarded as a Hilbert $C^{*}$-module over itself where the inner product is defined by $\langle a, b\rangle:=a^{*} b$. By $\mathbb{M}_{n}(\mathbb{C})$ we denote the $C^{*}$-algebra of all complex $n \times n$ matrices. We shall identify $\mathbb{B}\left(\mathbb{C}^{n}\right)$ and $\mathbb{M}_{n}(\mathbb{C})$ in the usual way. We refer the reader to $[11,17]$ for more information on Hilbert $C^{*}$-modules.

A concept of orthogonality in a Hilbert $\mathscr{A}$-module $\mathscr{X}$ can be defined with respect to the $\mathscr{A}$-valued inner product in a natural way, that is, two elements $x$ and $y$ of $\mathscr{X}$ are orthogonal, in short $x \perp y$, if $\langle x, y\rangle=0$. There are many different ways how one can extend this notion, see [3] and the references therein. One of them is the Birkhoff-James orthogonality: we say that $x$ and $y$ are Birkhoff-James orthogonal, and we write $x \perp_{B} y$, if $\|x\| \leq\|x+\lambda y\|$ for all $\lambda \in \mathbb{C}$. A well known characterization of the Birkhoff-James orthogonality is due to Arambašić and Rajić (see [2]):

$$
x \perp_{B} y \quad \Leftrightarrow \quad\left(\exists \varphi \in \Omega_{x}: \varphi(\langle x, y\rangle)=0\right) .
$$

It has been proved also by Bhattacharyya and Grover (cf. [5]). The statement of this nice characterization is so simple, and its existing proofs so extremely long, that one is easily seduced into an effort to find a simpler, shorter proof. The present work is the result of our attempt. It is worth mentioning that those four mathematicians applied a faithful representation $\pi: \mathscr{A} \rightarrow \mathbb{B}(\mathcal{H})$ (see [11, Theorem 2.6.1]) and linking algebra of $\mathscr{X}$ (see [17]). But we do not use this strong tool. We apply norm derivatives. In this paper we would like to present a simpler proof of this nice result and we demonstrate the power of the norm derivatives. We hope that it sheds new light on this intricate geometric structure of Hilbert $C^{*}$-modules and will provide the great applications of the mappings $\rho_{ \pm}$ in the future.

\section{Main Results: Norm derivatives in Hilbert $C^{*}$-Modules}

In this section, we first compute the norm derivatives in Hilbert $C^{*}$-modules. Then, as an application of our results, we get an explicit formula for the norm derivatives $\rho_{ \pm}$of certain elements in Hilbert $C^{*}$-modules. Moreover, we apply 
our results to give some solutions of the generalized Daugavet equation in the operator space $\mathbb{B}(\mathcal{H})$.

We start our work with the following lemma.

Lemma 2.1. [22] Suppose that $X$ is a real normed space. Let $D \subseteq X$ be a dense and star-shaped subset (i.e. $\alpha D \subseteq D$ for all $\alpha>0$ ). Let $M$ be a closed affine hyperplane (i.e. $\operatorname{codim} M=1)$ such that $0 \notin M$. Then $\operatorname{cl}(M \cap D)=M$.

It is worth mentioning that Lemma 2.1 played a significant role in the papers [22] and [23]. Its proof can be found in [22]. Here, this lemma will be a helpful tool, again.

We say that $X$ is smooth at point $x_{o}$ if there is a unique functional $f \in J\left(x_{o}\right)$. Now, we consider a set $\mathcal{N}_{s m}(X):=\{x \in X: X$ is smooth at $x\} \cup\{0\}$. In particular, we have

$$
x_{o} \in \mathcal{N}_{s m}(X) \Leftrightarrow \rho_{+}\left(x_{o}, \cdot\right)=\rho_{-}\left(x_{o}, \cdot\right)=\|x\| \operatorname{Re} f(\cdot), \quad f \in J\left(x_{o}\right) .
$$

It is known that if $\operatorname{dim} X<\infty$, then $\mathcal{N}_{s m}(X)$ is a dense, star-shaped subset of $X$ - cf. [22] or [1]. Thus we can rewrite Lemma 2.1 as

Lemma 2.2. Suppose that $Z$ is a two-dimensional real normed space. Let $\{x, y\} \subseteq$ $Z$ be a linearly independent subset. If we consider a line $M$ spanned by the vectors $x, y$ (i.e. $M:=\{x+t y \in Z: t \in \mathbb{R}\})$, then $\operatorname{cl}\left(M \cap \mathcal{N}_{s m}(Z)\right)=M$.

We are now in a position to prove the main result of this paper.

Theorem 2.3. Let $\mathscr{X}$ be a Hilbert $\mathscr{A}$-module, and $x, y \in \mathscr{X}$. Then

$$
\rho_{+}(x, y)=\max \left\{\operatorname{Re} \varphi(\langle x, y\rangle): \varphi \in \Omega_{x}\right\} .
$$

Proof. We may and shall assume that $x \neq 0$ otherwise the statement trivially holds. Let $\varphi \in \Omega_{x}$, that is, $\varphi \in \mathcal{S}(\mathscr{A})$ and $\varphi(\langle x, x\rangle)=\|x\|^{2}$. It is easy to check that $\frac{1}{\|x\|} \varphi(\langle x, \cdot\rangle) \in J(x)$. Thus the property (1.1) yields $\|x\| \operatorname{Re} \frac{1}{\|x\|} \varphi(\langle x, y\rangle) \leq$ $\rho_{+}^{\prime}(x, y)$. Hence $\operatorname{Re} \varphi(\langle x, y\rangle) \leq \rho_{+}(x, y)$. Passing to the supremum over $\varphi \in \Omega_{x}$ we get

$$
\sup \left\{\operatorname{Re} \varphi(\langle x, y\rangle): \varphi \in \Omega_{x}\right\} \leq \rho_{+}(x, y)
$$

To complete the proof, we must find $\varphi \in \Omega_{x}$ such that $\operatorname{Re} \varphi(\langle x, y\rangle)=\rho_{+}(x, y)$. Then we will able to write "max" instead of "sup". We consider two cases. First assume that $x$ and $y$ are linearly dependent, i.e. $y=\alpha x$ with some number $\alpha$. Fix $\varphi \in \Omega_{x}$. Then

$$
\begin{aligned}
\rho_{+}(x, y) & =\rho_{+}(x, \alpha x) \stackrel{(\mathrm{P} 3)}{=} \operatorname{Re}\left(\alpha\|x\|^{2}\right)=\operatorname{Re}(\alpha \varphi(\langle x, x\rangle)) \\
& =\operatorname{Re} \varphi(\langle x, \alpha x\rangle)=\operatorname{Re} \varphi(\langle x, y\rangle) .
\end{aligned}
$$

So, the first case is complete. Now, suppose that $\{x, y\}$ is a linearly independent subset. Let us define a real subspace $Z \subseteq \mathscr{X}$ by the formula

$$
Z:=\{\alpha x+\beta y \in \mathscr{X}: \alpha, \beta \in \mathbb{R}\} .
$$


Put $M:=\{x+t y \in Z: t \in \mathbb{R}\}$. It follows from Lemma 2.2 that $\operatorname{cl}(M \cap$ $\left.\mathcal{N}_{s m}(Z)\right)=M$. This equality yields, in particular, that there exists a sequence $\left\{t_{n}: n=1,2, \ldots\right\}$ in $(0,+\infty)$ such that $t_{n} \rightarrow 0^{+}$and $x+t_{n} y \in \mathcal{N}_{s m}(Z)$.

Let us define for the moment a closed subalgebra $\mathscr{A}_{0} \subseteq \mathscr{A}$ spanned by the vectors $\langle x, x\rangle,\langle y, y\rangle,\langle x, y\rangle$ and $\langle y, x\rangle$. In particular, we have

$$
\left\langle x+t_{n} y, x+t_{n} y\right\rangle,\left\langle x+t_{n} y, y\right\rangle \in \mathscr{A}_{0} .
$$

Moreover, it is easily seen that $\mathscr{A}_{0}$ is separable. Also, there exist states $\varphi_{1}, \varphi_{2}, \varphi_{3}, \ldots \in$ $\mathcal{S}\left(\mathscr{A}_{0}\right)$ such that

$$
\varphi_{n}\left(\left\langle x+t_{n} y, x+t_{n} y\right\rangle\right)=\left\|x+t_{n} y\right\|^{2} .
$$

Now our attention must focus on the real two-dimensional space $Z$. Fix $n \in \mathbb{N}$. It is not difficult to see that $\left.\frac{1}{\left\|x+t_{n} y\right\|} \operatorname{Re} \varphi_{n}\left(\left\langle x+t_{n} y, \cdot\right\rangle\right)\right|_{Z}: Z \rightarrow \mathbb{R}$ is a $\mathbb{R}$-linear functional and

$$
\left.\left.\frac{1}{\left\|x+t_{n} y\right\|} \operatorname{Re} \varphi_{n}\left(\left\langle x+t_{n} y, \cdot\right\rangle\right)\right|_{Z} \in J\left(x+t_{n} y\right)\right|_{Z}
$$

The space $Z$ is smooth at $x+t_{n} y$. Thus, from (2.1) and (2.3) it is known that

$$
\rho_{+}\left(x+t_{n} y, y\right)=\operatorname{Re} \varphi_{n}\left(\left\langle x+t_{n} y, y\right\rangle\right) .
$$

By Alaoglu's Theorem, we known that the closed unit ball $B_{\mathscr{A}_{0}^{*}}$ is weakly* compact. It is easy to check that subset $\mathcal{S}\left(\mathscr{A}_{0}\right) \subseteq \mathscr{A}_{0}^{*}$ is a norm-closed convex subset of the weakly* compact ball of $B_{\mathscr{A}_{0}^{*}}$. Therefore $\mathcal{S}\left(\mathscr{A}_{0}\right)$ is weakly* compact. Since $\mathscr{A}_{0}$ is separable, $\mathcal{S}\left(\mathscr{A}_{0}\right)$ is weakly* sequentially compact. Thus, there are an element $\varphi_{o} \in B_{\mathscr{A}_{o}^{*}}$ and a subsequence $\left(\varphi_{n_{k}}\right)_{k=1}^{\infty} \subseteq B_{\mathscr{A}_{o}^{*}}$ such that $\varphi_{n_{k}} \stackrel{w *}{\longrightarrow} \varphi_{o}$. Since $\left\langle x+t_{n_{k}} y, y\right\rangle \stackrel{\|\cdot\|}{\longrightarrow}\langle x, y\rangle$, we conclude that

$$
\operatorname{Re} \varphi_{n_{k}}\left(\left\langle x+t_{n_{k}} y, y\right\rangle\right) \longrightarrow \operatorname{Re} \varphi_{o}(\langle x, y\rangle)
$$

Similarly, since $\left\langle x+t_{n_{k}} y, x+t_{n_{k}} y\right\rangle \stackrel{\|\cdot\|}{\longrightarrow}\langle x, x\rangle$, it follows that

$$
\varphi_{n_{k}}\left(\left\langle x+t_{n_{k}} y, x+t_{n_{k}} y\right\rangle\right) \longrightarrow \varphi_{o}(\langle x, x\rangle) .
$$

Combining the three conditions (P5)-(2.4)-(2.5) we deduce that

$$
\operatorname{Re} \varphi_{o}(\langle x, y\rangle)=\rho_{+}(x, y) .
$$

Furthermore, combining (2.2) with (2.6) yields $\varphi_{o}(\langle x, x\rangle)=\|x\|^{2}$.

Now we back to the $C^{*}$-algebra $\mathscr{A}$ and we go to the dual normed space $\mathscr{A}^{*}$. Namely, since $\varphi_{0}: \mathscr{A}_{0} \rightarrow \mathbb{C}$ is a state on $\mathscr{A}_{0}$, it follows that there exists a state $\varphi$ on $\mathscr{A}$ such that $\left.\varphi\right|_{\mathscr{A}_{0}}=\varphi_{o}$ (see e.g. [10, p.259]). From this we get $\operatorname{Re} \varphi(\langle x, y\rangle)=$ $\rho_{+}(x, y)$ and $\varphi(\langle x, x\rangle)=\|x\|^{2}$. The proof is completed.

Now we are able to calculate a formula for the norm derivative $\rho_{-}$in Hilbert $C^{*}$-modules.

Theorem 2.4. Let $\mathscr{X}$ be a Hilbert $\mathscr{A}$-module, and $x, y \in \mathscr{X}$. Then

$$
\rho_{-}(x, y)=\min \left\{\operatorname{Re} \varphi(\langle x, y\rangle): \varphi \in \Omega_{x}\right\} .
$$


Proof. Applying (P2) and Theorem 2.3 we get

$$
\begin{aligned}
\rho_{-}(x, y) & =-\rho_{+}(x,-y)=-\max \left\{\operatorname{Re} \varphi(\langle x,-y\rangle): \varphi \in \Omega_{x}\right\} \\
& =\min \left\{\operatorname{Re} \varphi(\langle x, y\rangle): \varphi \in \Omega_{x}\right\},
\end{aligned}
$$

and we are done.

Recall that every $C^{*}$-algebra $\mathscr{A}$ can be regarded as a Hilbert $C^{*}$-module over itself with the inner product $\langle a, b\rangle:=a^{*} b$. Thus, as a consequence of Theorem 2.3 (and Theorem 2.4) we have the following result.

Corollary 2.5. Let $\mathscr{A}$ be a $C^{*}$-algebra, and $a, b \in \mathscr{A}$. Then

$$
\begin{aligned}
& \rho_{+}(a, b)=\max \left\{\operatorname{Re} \varphi\left(a^{*} b\right): \varphi \in \Omega_{a}\right\}, \\
& \rho_{-}(a, b)=\min \left\{\operatorname{Re} \varphi\left(a^{*} b\right): \varphi \in \Omega_{a}\right\} .
\end{aligned}
$$

Let $\mathbb{T}(\mathcal{H})$ be trace-class operators on a Hilbert space $(\mathcal{H},[\cdot, \cdot])$. It is well known that (see e.g. [20, Theorem 4.2.1]) every state $\varphi$ of $\mathbb{K}(\mathcal{H})$ is of the form $a \rightarrow \operatorname{tr}(p a)$ for some positive trace one operator $p \in \mathbb{T}(\mathcal{H})$. Therefore, as an immediate consequence of Theorem 2.3 and Theorem 2.4, we have the following result.

Proposition 2.6. Let $\mathscr{X}$ be a Hilbert $\mathbb{K}(\mathcal{H})$-module, and $x, y \in \mathscr{X}$. Then

$$
\begin{aligned}
& \rho_{+}(x, y)=\max \left\{\operatorname{Retr}(p\langle x, y\rangle): p \in \mathbb{P}(\mathcal{H}), \operatorname{tr}(p\langle x, x\rangle)=\|x\|^{2}\right\}, \\
& \rho_{-}(x, y)=\min \left\{\operatorname{Retr}(p\langle x, y\rangle): p \in \mathbb{P}(\mathcal{H}), \operatorname{tr}(p\langle x, x\rangle)=\|x\|^{2}\right\},
\end{aligned}
$$

where $\mathbb{P}(\mathcal{H})=\{p \in \mathbb{T}(\mathcal{H}): p$ is positive trace one $\}$.

We continue this section by applying our results to get an explicit formula for the norm derivatives $\rho_{ \pm}$of certain elements in Hilbert $C^{*}$-modules.

Theorem 2.7. Let $\mathscr{X}$ be a Hilbert $\mathscr{A}$-module, and $x \in \mathscr{X}$. Then

$$
\rho_{+}(x, x\langle x, x\rangle)=\|x\|^{4}=\rho_{-}(x, x\langle x, x\rangle) .
$$

Proof. Fix $\varphi \in \mathcal{S}(\mathscr{A})$ such that $\varphi(\langle x, x\rangle)=\|x\|^{2}$. We may suppose that $\mathscr{A}$ is a Banach algebra with identity (by going to extensions $\tilde{\mathscr{A}}$ and $\tilde{\varphi}=\left.\varphi\right|_{\mathscr{A}}$ if necessary; see $[10$, pp.194, 259]). So, let $e$ be the identity of $\mathscr{A}$. Then

$$
\|x\|^{4}=|\varphi(\langle x, x\rangle e)|^{2} \stackrel{(1.2)}{\leq} \varphi\left(\langle x, x\rangle^{2}\right) \varphi\left(e^{2}\right) \leq \varphi\left(\langle x, x\rangle^{2}\right) \leq\left\|\langle x, x\rangle^{2}\right\| \leq\|x\|^{4} .
$$

This implies

$$
\varphi\left(\langle x, x\rangle^{2}\right)=\|x\|^{4} .
$$

Therefore we obtain the following equalities

$$
\begin{aligned}
\operatorname{Re} \varphi\left(\left\langle x,\|x\|^{2} x-x\langle x, x\rangle\right\rangle\right) & =\|x\|^{2} \operatorname{Re} \varphi(\langle x, x\rangle)-\operatorname{Re} \varphi(\langle x, x\langle x, x\rangle\rangle) \\
& =\|x\|^{2} \operatorname{Re}\|x\|^{2}-\operatorname{Re} \varphi(\langle x, x\rangle\langle x, x\rangle) \\
& \stackrel{(2.8)}{=}\|x\|^{2}\|x\|^{2}-\|x\|^{4}=0 .
\end{aligned}
$$


Now we apply Theorem 2.3 (resp. Theorem 2.4). Namely, since $\varphi$ was arbitrarily chosen from $\Omega_{x}$, passing to the maximum (resp. minimum) over $\varphi \in \Omega_{x}$, we get

$$
\rho_{+}\left(x,\|x\|^{2} x-x\langle x, x\rangle\right)=0 \quad \text { and } \quad \rho_{-}\left(x,\|x\|^{2} x-x\langle x, x\rangle\right)=0 .
$$

So, from (P3) and (P2) we obtain, respectively, $\|x\|^{2} \cdot\|x\|^{2}-\rho_{-}(x,\langle x, x\rangle)=0$ and $\|x\|^{2} \cdot\|x\|^{2}-\rho_{+}(x, x\langle x, x\rangle)=0$, and we may consider (2.7) as shown.

An element $x$ in a normed space $(X,\|\cdot\|)$ is called norm-parallel to another element $y \in X$ (see [30] and the references therein), denoted by $x \| y$, if $\|x+\xi y\|=$ $\|x\|+\|y\|$ for some complex unit $\xi$. In the framework of inner product spaces, the norm-parallel relation is exactly the usual vectorial parallel relation, that is, $x \| y$ if and only if $x$ and $y$ are linearly dependent. In the setting of normed linear spaces, two linearly dependent vectors are norm-parallel, but the converse is false in general. Many characterizations of the norm-parallelism for operators spaces $\mathbb{B}(X, Y)$ and elements of an arbitrary Hilbert $C^{*}$-module were given in [7], [25], [30] and [31].

Theorem 2.8. Suppose that $\mathscr{X}$ is a Hilbert $\mathscr{A}$-module, and let $x \in \mathscr{X}, \alpha, \beta \in$ $(0,+\infty)$. Then

$$
\|\alpha x+\beta x\langle x, x\rangle\|=\alpha\|x\|+\beta\|x\|^{3} .
$$

In particular, $x$ is norm-parallel to $x\langle x, x\rangle$.

Proof. It is clear that we may assume $x \neq 0$. Then we have

$$
\begin{aligned}
\alpha\|x\|^{2}+\beta\|x\|^{4} & \stackrel{(2.7)}{=} \alpha\|x\|^{2}+\beta \rho_{+}(x, x\langle x, x\rangle) \stackrel{(\mathrm{P} 3, \mathrm{P} 4)}{=} \rho_{+}(x, \alpha x+\beta x\langle x, x\rangle) \\
& \stackrel{(\mathrm{P} 1)}{\leq}\|x\| \cdot\|\alpha x+\beta x\langle x, x\rangle\| \leq\|x\| \cdot(\alpha\|x\|+\beta\|x\langle x, x\rangle\|) \\
& \leq\|x\| \cdot(\alpha\|x\|+\beta\|x\| \cdot\|\langle x, x\rangle\|)=\alpha\|x\|^{2}+\beta\|x\|^{4} .
\end{aligned}
$$

Thus the string of inequalities becomes a string of equalities and we obtain $\alpha\|x\|^{2}+\beta\|x\|^{4}=\|x\| \cdot\|\alpha x+\beta x\langle x, x\rangle\|$. Dividing by $\|x\|$, we have (2.9).

In the context of bounded linear operators on normed spaces, the well-known Daugavet equation $\|I+T\|=1+\|T\|$ is a particular case of parallelism. We refer to the book [21] and more recent the paper [27] for motivations, history, various aspects and problems connected with the Daugavet equation. It is worth mentioning that a generalized Daugavet equation $\|T+S\|=\|T\|+\|S\|$ is one useful property in solving a variety of problems in approximation theory (cf. [27]).

A consequence of Theorem 2.8 is established in the next result.

Corollary 2.9. Let $T \in \mathbb{B}(\mathcal{H})$. Then

$$
\left\|T+T T^{*} T\right\|=\|T\|+\|T\|^{3} .
$$

Moreover, if $\operatorname{dist}(T, \mathbb{K}(\mathcal{H}))<\|T\|$ or $\operatorname{dist}\left(T T^{*} T, \mathbb{K}(\mathcal{H})\right)<\left\|T T^{*} T\right\|$, then there exists a unit vector $x_{o} \in \mathcal{H}$ such that $\frac{T}{\|T\|} x_{o}=\frac{T T^{*} T}{\left\|T T^{*} T\right\|} x_{o},\left\|T x_{o}\right\|=\|T\|$ and $\left\|T T^{*} T x_{o}\right\|=\left\|T T^{*} T\right\|$. 
Proof. It follows from (2.9) that $\left\|T+T T^{*} T\right\|=\|T\|+\|T\|^{3}$. In addition, it is not difficult to check that $\left\|T T^{*} T\right\|=\|T\|^{3}$. Therefore both $T$ and $T T^{*} T$ satisfy the generalized Daugavet equation

$$
\left\|T+T T^{*} T\right\|=\|T\|+\left\|T T^{*} T\right\| .
$$

Now, suppose that $\operatorname{dist}(T, \mathbb{K}(\mathcal{H}))<\|T\|$ or $\operatorname{dist}\left(T T^{*} T, \mathbb{K}(\mathcal{H})\right)<\left\|T T^{*} T\right\|$. Recall that $\mathbb{K}(\mathcal{H})$ is an $M$-ideal in $\mathbb{B}(\mathcal{H})$ (see [25]). Moreover, $\mathcal{H}$ is strictly convex. Thus these information are legitimates to apply [27, Theorem 4.4]. So, it follows from [27, Theorem 4.4] and (2.10) that there exists a unit vector $x_{o} \in \mathcal{H}$ such that $\frac{T}{\|T\|} x_{o}=\frac{T T^{*} T}{\left\|T T^{*} T\right\|} x_{o},\left\|T x_{o}\right\|=\|T\|$ and $\left\|T T^{*} T x_{o}\right\|=\left\|T T^{*} T\right\|$.

\section{An application: Orthogonalities in Hilbert $C^{*}$-modules}

In a normed linear space $(X,\|\cdot\|)$, for two vectors $x, y \in X$, one can consider the Birkhoff-James orthogonality (see [6, 13]) defined by

$$
x \perp_{B} y \quad: \Leftrightarrow \quad \forall \forall_{\lambda \in \mathbb{C}}\|x\| \leq\|x+\lambda y\| .
$$

We will consider also the $r$-Birkhoff-James orthogonality, defined by

$$
x \perp_{B}^{r} y \quad: \Leftrightarrow \quad \forall_{\alpha \in \mathbb{R}}\|x\| \leq\|x+\alpha y\| .
$$

Notice that $\perp_{B} \subseteq \perp_{B}^{r}$, but the converse is false in general. For example, let us take $X=\mathbb{C}^{2}$ and let $x=(1,0), y=(i, 0)$. Then for all $\alpha \in \mathbb{R}$ we have

$$
\|x+\alpha y\|=\|(1+\alpha i, 0)\|=\sqrt{1+\alpha^{2}} \geq 1=\|x\| .
$$

Hence $x \perp_{B}^{r} y$. But $x \not \perp_{B} y$ since for $\lambda:=i$ we have $\|x+\lambda y\|=0<1=\|x\|$.

We will use the following characterizations of both orthogonality relations. Namely, for arbitrary $x, y \in X$, we have (see [1, 14]):

$$
x \perp_{B} y \quad \Leftrightarrow \quad \inf _{0 \leq \theta<2 \pi} \rho_{+}\left(x, e^{i \theta} y\right) \geq 0
$$

and

$$
x \perp_{B}^{r} y \quad \Leftrightarrow \quad \rho_{-}(x, y) \leq 0 \leq \rho_{+}(x, y) .
$$

When $X=\mathbb{M}_{n}(\mathbb{C})$ and $T, S \in X$, a very tractable condition of the BirkhoffJames orthogonality was found by Bhatia and Šemrl in [4]. They showed that $T \perp_{B} S$ if and only if there exists a unit vector $x \in \mathbb{C}^{n}$ such that

$$
\|T x\|=\|T\| \quad \text { and } \quad[T x, S x]=0 .
$$

Later Bhattacharyya and Grover [5] showed that $T \perp_{B}^{r} S$ if and only if there exists a unit vector $x \in \mathbb{C}^{n}$ such that

$$
\|T x\|=\|T\| \quad \text { and } \quad \operatorname{Re}[T x, S x]=0 .
$$

To summarize, the papers [4], [5] and conditions (3.3), (3.4) motivate the next theorem. In other words, we now obtain a characterization of real version of the Birkhoff-James orthogonality in Hilbert $C^{*}$-modules in terms of states of the underlying $C^{*}$-algebra.

Theorem 3.1. Let $\mathscr{X}$ be a Hilbert $\mathscr{A}$-module, and $x, y \in \mathscr{X}$. The following statements are equivalent: 
(i) $x \perp_{B}^{r} y$,

(ii) there exists $\varphi \in \Omega_{x}$ such that $\operatorname{Re} \varphi(\langle x, y\rangle)=0$.

Proof. We assume that $x \neq 0$ (otherwise the result is trivial). First, suppose that $x \perp_{B}^{r} y$. By (3.2) we have $\rho_{-}(x, y) \leq 0 \leq \rho_{+}(x, y)$. So, by Theorem 2.3 , there exist $\varphi_{1}, \varphi_{2} \in \Omega_{x}$ such that

$$
\operatorname{Re} \varphi_{1}(\langle x, y\rangle) \leq 0 \leq \operatorname{Re} \varphi_{2}(\langle x, y\rangle) .
$$

It follows from the above inequality that for some $\lambda_{o} \in[0,1]$ we have

$$
\lambda_{o} \operatorname{Re} \varphi_{1}(\langle x, y\rangle)+\left(1-\lambda_{o}\right) \operatorname{Re} \varphi_{2}(\langle x, y\rangle)=0 .
$$

Since $S(\mathscr{A})$ is convex, $\lambda_{o} \varphi_{1}+\left(1-\lambda_{o}\right) \varphi_{2} \in S(\mathscr{A})$. Put $\varphi:=\lambda_{o} \varphi_{1}+\left(1-\lambda_{o}\right) \varphi_{2}$. We get then $\varphi \in S(\mathscr{A})$ and $\varphi(\langle x, x\rangle)=\|x\|^{2}$. Hence $\varphi \in \Omega_{x}$. Also, by (3.5), $\operatorname{Re} \varphi(\langle x, y\rangle)=0$.

Now we prove the implication (ii) $\Rightarrow(\mathrm{i})$. Assume that there exists $\varphi \in \Omega_{x}$ such that $\operatorname{Re} \varphi(\langle x, y\rangle)=0$. Fix arbitrarily $\alpha \in \mathbb{R}$. We have

$$
\begin{aligned}
\|x\|^{2} & =\varphi(\langle x, x\rangle)=\operatorname{Re} \varphi(\langle x, x\rangle)+\alpha \operatorname{Re} \varphi(\langle x, y\rangle) \\
& =\operatorname{Re} \varphi(\langle x, x+\alpha y\rangle) \leq|\varphi(\langle x, x+\alpha y\rangle)| \\
& \leq\|\langle x, x+\alpha y\rangle\| \leq\|x\| \cdot\|x+\alpha y\| .
\end{aligned}
$$

Thus $\|x\|^{2} \leq\|x\| \cdot\|x+\alpha y\|$ and so $\|x\| \leq\|x+\alpha y\|$. Hence $x \perp_{B}^{r} y$.

Finally, we are able to give a simple proof of (1.3) using the map $\rho_{+}$, i.e. Theorem 2.3. Let us recall again: unlike [2, 5], we did not apply a faithful representation $\pi: \mathscr{A} \rightarrow \mathbb{B}(\mathcal{H})$ and linking algebra of $\mathscr{X}$. Therefore the proofs of Theorem 2.3 and Theorem 3.2 are simpler and shorter than the proofs in $[2,5]$. So, we now show that there is another (and easier) way to get the celebrated result (1.3).

Theorem 3.2. Let $\mathscr{X}$ be a Hilbert $\mathscr{A}$-module, and $x, y \in \mathscr{X}$. The following statements are mutually equivalent:

(i) $x \perp_{B} y$,

(ii) there exists $\varphi \in \Omega_{x}$ such that $\varphi(\langle x, y\rangle)=0$,

(iii) $\|x+\lambda y\|^{2} \geq\|x\|^{2}+|\lambda|^{2} m(y)$ for all $\lambda \in \mathbb{C}$,

where $m(y):=\inf \{\varphi(\langle y, y\rangle): \varphi \in \mathcal{S}(\mathscr{A})\}$.

Proof. (i) $\Rightarrow$ (ii) Let $x \perp_{B} y$. Then, by Theorem 2.3 and (3.1), we get

$$
\inf _{0 \leq \theta<2 \pi} \max \left\{\operatorname{Re} e^{i \theta} \varphi(\langle x, y\rangle): \varphi \in \Omega_{x}\right\} \geq 0 .
$$

It is easy to see that the set $E:=\left\{\varphi(\langle x, y\rangle): \varphi \in \Omega_{x}\right\}$ is convex and hence its closure is a closed convex set. Therefore, by (3.6), the set $E$ has such a position in the complex plane that it must contain at least one value with positive real part, under all rotations around the origin. Thus $E$ must contain zero, and so there is a $\varphi \in \Omega_{x}$ such that $\varphi(\langle x, y\rangle)=0$. 
(ii) $\Rightarrow$ (iii) Suppose (ii) holds. Then, for every $\lambda \in \mathbb{C}$, we have

$$
\begin{aligned}
\|x+\lambda y\|^{2} & \geq \varphi(\langle x+\lambda y, x+\lambda y\rangle) \\
& =\varphi(\langle x, x\rangle)+2 \operatorname{Re}(\lambda \varphi(\langle x, y\rangle))+|\lambda|^{2} \varphi(\langle y, y\rangle) \\
& =\|x\|^{2}+|\lambda|^{2} \varphi(\langle y, y\rangle) .
\end{aligned}
$$

Therefore

$$
\|x+\lambda y\|^{2} \geq\|x\|^{2}+|\lambda|^{2} \varphi(\langle y, y\rangle)
$$

which yields $\|x+\lambda y\|^{2} \geq\|x\|^{2}+|\lambda|^{2} m(y)$ for all $\lambda \in \mathbb{C}$.

(iii) $\Rightarrow$ (i) The implication is trivial.

As a natural generalization of the notion of Birkhoff--James orthogonality, the concept of strong Birkhoff-James orthogonality, which involves modular structure of a Hilbert $C^{*}$-module was introduced in [3]. When $x$ and $y$ are elements of a Hilbert $\mathscr{A}$-module $\mathscr{X}$, we consider the strong Birkhoff-James orthogonality:

$$
x \perp_{B}^{s} y \quad: \Leftrightarrow \quad \forall a \in \mathscr{A}\|x\| \leq\|x+y a\| .
$$

One can easily observe that $x \perp y \Longrightarrow x \perp_{B}^{s} y \Longrightarrow x \perp_{B}^{r} y$, while the converses do not hold in general (see [3]).

In the next result we establish characterizations of the strong Birkhoff-James orthogonality for elements of a Hilbert $C^{*}$-module based on norm derivatives. We will apply our new tools - Theorems 2.3, 2.4 and 3.1.

Theorem 3.3. Let $\mathscr{X}$ be a Hilbert $\mathscr{A}$-module, and $x, y \in \mathscr{X}$. The following statements are mutually equivalent:

(i) $x \perp_{B}^{s} y$,

(ii) $\rho_{-}(x, y a) \leq 0$ for all $a \in \mathscr{A}$,

(iii) $\rho_{+}(x, y a) \geq 0$ for all $a \in \mathscr{A}$.

Proof. (i) $\Rightarrow$ (ii) Let $x \perp_{B}^{s} y$. Then $x \perp_{B}^{r} y\langle y, x\rangle$. So, by Theorem 3.1, there exists $\varphi_{o} \in \Omega_{x}$ such that $\operatorname{Re} \varphi_{o}(\langle x, y\langle y, x\rangle\rangle)=0$. Hence $\operatorname{Re} \varphi_{o}(\langle x, y\rangle\langle y, x\rangle)=0$. Thus $\varphi_{o}(\langle x, y\rangle\langle y, x\rangle)=0$, since $\varphi_{o}(\langle x, y\rangle\langle y, x\rangle) \in \mathbb{R}$. Therefore,

$$
\left|\operatorname{Re} \varphi_{o}(\langle x, y a\rangle)\right|^{2} \leq\left|\varphi_{o}(\langle x, y\rangle a)\right|^{2} \stackrel{(1.2)}{\leq} \varphi_{o}(\langle x, y\rangle\langle y, x\rangle) \varphi_{o}\left(a^{*} a\right)=0
$$

for each $a \in \mathscr{A}$. This implies $\operatorname{Re} \varphi_{o}(\langle x, y a\rangle)=0$. Hence

$$
\min \left\{\operatorname{Re} \varphi(\langle x, y a\rangle): \varphi \in \Omega_{x}\right\} \leq 0
$$

and by Theorem 2.4 it follows that $\rho_{-}(x, y a) \leq 0$ for all $a \in \mathscr{A}$.

(ii) $\Rightarrow$ (iii) By the condition (ii), $\rho_{-}(x, y(-a)) \leq 0$ for all $a \in \mathscr{A}$. Hence, by (P2), $\rho_{+}(x, y a)=-\rho_{-}(x, y(-a)) \geq 0$ for all $a \in \mathscr{A}$.

(iii) $\Rightarrow$ (i) Suppose (iii) holds. We may assume that $x \neq 0$ otherwise (i) trivially holds. So, fix $a \in \mathscr{A}$. By Theorem 2.3, there exists a state $\varphi_{a}$ in $\Omega_{x}$ such that 
$\operatorname{Re} \varphi_{a}(\langle x, y a\rangle) \geq 0$. Then we have

$$
\begin{aligned}
0 & \leq \operatorname{Re} \varphi_{a}(\langle x, y a\rangle)=\operatorname{Re} \varphi_{a}(\langle x, x+y a\rangle)-\operatorname{Re} \varphi_{a}(\langle x, x\rangle) \\
& \leq\left|\varphi_{a}(\langle x, x+y a\rangle)\right|-\|x\|^{2} \leq\|\langle x, x+y a\rangle\|-\|x\|^{2} \\
& \leq\|x\| \cdot\|x+y a\|-\|x\|^{2}=\|x\| \cdot(\|x+y a\|-\|x\|) .
\end{aligned}
$$

This implies that $\|x\| \leq\|x+y a\|$. Hence $x \perp_{B}^{s} y$.

We recall that (see $[12,18])$ two elements $x$ and $y$ of a normed linear space $X$ are $\rho$-orthogonal if $\rho(x, y):=\frac{\rho_{+}(x, y)+\rho_{-}(x, y)}{2}=0$, and in this case we write $x \perp_{\rho} y$. It is worth mentioning that the notion of $\rho$-orthogonality may be a strong tool. Indeed, the open problem posed in [1] was solved in in the paper [29] and the concept of $\rho$-orthogonality played a significant role. For facts about the $\rho$-orthogonality in normed linear spaces, we refer the reader to $[8,9,19,32]$.

If $x$ and $y$ are elements of a Hilbert $\mathscr{A}$-module $\mathscr{X}$, then we have

$$
x \perp y \Longrightarrow x \perp_{\rho} y \Longrightarrow x \perp_{B}^{r} y .
$$

Indeed, if $x \perp y$, then $\langle x, y\rangle=0$. Thus, for every $\varphi \in \Omega_{x}$, we have $\operatorname{Re} \varphi(\langle x, y\rangle)=0$ and so by Theorem 2.3 and Theorem 2.4 we obtain $\rho_{-}(x, y)=\rho_{+}(x, y)=0$. Hence $\rho(x, y)=0$, and thus $x \perp_{\rho} y$.

Further, if $x \perp_{\rho} y$, then $\rho_{-}(x, y)+\rho_{+}(x, y)=0$ and therefore $\rho_{-}(x, y) \leq 0 \leq$ $\rho_{+}(x, y)$ by $(\mathrm{P} 1)$. It follows (3.2) from that $x \perp_{B}^{r} y$.

As an immediate consequence of Theorem 2.3 and Theorem 2.4 we obtain a characterization of the $\rho$-orthogonality in Hilbert $C^{*}$-modules as follows.

Theorem 3.4. Let $\mathscr{X}$ be a Hilbert $\mathscr{A}$-module, and $x, y \in \mathscr{X}$. The following statements are equivalent:

(i) $x \perp_{\rho} y$,

(ii) $\max \left\{\operatorname{Re} \varphi(\langle x, y\rangle): \varphi \in \Omega_{x}\right\}=\max \left\{-\operatorname{Re} \varphi(\langle x, y\rangle): \varphi \in \Omega_{x}\right\}$.

Remark 3.5. Notice that the converses in (3.7) do not hold in general. For example, consider $\mathbb{M}_{2}(\mathbb{C})$ as a Hilbert $\mathbb{M}_{2}(\mathbb{C})$-module and let $T=\left[\begin{array}{ll}1 & 0 \\ 0 & 1\end{array}\right], S=\left[\begin{array}{cc}-1 & 0 \\ 0 & 1\end{array}\right]$ and $R=\left[\begin{array}{cc}-1 & 0 \\ 0 & 0\end{array}\right]$. Then simple computations show that

$$
\rho_{+}(T, S)=-\rho_{-}(T, S)=-\rho_{-}(T, R)=1 \quad \text { and } \quad \rho_{+}(T, R)=0 .
$$

Hence $T \perp_{\rho} S$. But $T \not \perp S$, since $\langle T, S\rangle=S \neq 0$. Also, $T \not \perp_{\rho} R$ but

$$
\|T+\alpha R\|=\left\|\left[\begin{array}{cc}
1-\alpha & 0 \\
0 & 1
\end{array}\right]\right\|=\max \{|1-\alpha|, 1\} \geq 1=\|T\|
$$

for all $\alpha \in \mathbb{R}$. Therefore $T \perp_{B}^{r} R$.

To end the work we show that the orthogonalities $\perp_{\rho}$ and $\perp_{B}^{s}$ are incomparable. Indeed, since for $C=\left[\begin{array}{cc}1 & 0 \\ 0 & -1\end{array}\right]$ we have $\|T+S C\|=0<1=\|T\|$, we get 
$T \not \perp_{B}^{s} S$. Furthermore, for every $A=\left[\begin{array}{ll}a & b \\ c & d\end{array}\right] \in \mathbb{M}_{2}(\mathbb{C})$ we have $\|T+R A\|=$ $\left\|\left[\begin{array}{cc}1-a & -b \\ 0 & 1\end{array}\right]\right\| \geq 1=\|T\|$, whence $T \perp_{B}^{s} R$. Therefore $\perp_{\rho} \nsubseteq \perp_{B}^{s}$ and $\perp_{\rho} \nsupseteq \perp_{B}^{s}$.

\section{REFERENCES}

1. C. Alsina, J. Sikorska and M. S. Tomás, Norm Derivatives and Characterizations of Inner Product Spaces, World Scientific, Hackensack, NJ, 2010.

2. Lj. Arambašić and R. Rajić, The Birkhoff-James orthogonality in Hilbert $C^{*}$-modules, Linear Algebra Appl. 437 (2012), 1913-1929.

3. Lj. Arambašić and R. Rajić, A strong version of the Birkhoff-James orthogonality in Hilbert $C^{*}$-modules, Ann. Funct. Anal. 5 (2014), no. 1, 109-120.

4. R. Bhatia and P. Šemrl, Orthogonality of matrices and some distance problems, Linear Algebra Appl. 287(1-3) (1999), 77-85.

5. T. Bhattacharyya and P. Grover, Characterization of Birkhoff-James orthogonality, J. Math. Anal. Appl. 407 (2013), no. 2, 350-358.

6. G. Birkhoff, Orthogonality in linear metric spaces, Duke Math. J. 1 (1935), 169-172.

7. T. Bottazzi, C. Conde, M. S. Moslehian, P. Wójcik and A. Zamani, Orthogonality and parallelism of operators on various Banach spaces, J. Aust. Math. Soc. 106 (2019), 160183.

8. J. Chmieliński and P. Wójcik, On a $\rho$-orthogonality, Aequationes Math. 80 (2010), 45-55.

9. J. Chmieliński and P. Wójcik, $\rho$-orthogonality and its preservation-revisited, Recent Developments in Functional Equation and Inequalities, Banach Center Publ., Volume 99, Institute of Mathematic, Polish Academy of Sciences, Warszawa 2013, 17-30.

10. J. B. Conway, A Course in Functional Analysis, Springer-Verlag New York Inc., 1985.

11. J. Dixmier, $C^{*}$-Algebras, North-Holland, Amsterdam, 1981.

12. S. S. Dragomir, Semi-Inner Products and Applications, Nova Science Publishers Inc, Hauppauge (2004).

13. R. C. James, Orthogonality in normed linear spaces, Duke Math. J. 12 (1945), 291-302.

14. D. J. Keckić, Orthogonality in $\mathcal{C}_{1}$ and $\mathcal{C}_{\infty}$ spaces and normal derivations, J. Operator Theory 51 (2004), 89-104.

15. D. J. Keckić, Gateaux derivative of $B(H)$ norm, Proc. Amer. Math. Soc. 133 (2005), 20612067.

16. D. J. Keckić, Orthogonality and smooth points in $C(K)$ and $C_{b}(\Omega)$, Eurasian Math. J. 3 (2012), no. 4, 44-52.

17. V. M. Manuilov and E. V. Troitsky, Hilbert $C^{*}$-modules, In: Translations of Mathematical Monographs. 226, American Mathematical Society, Providence, RI, 2005.

18. P. M. Miličić, Sur la G-orthogonalité dans les espéaceésnormés, Math. Vesnik. 39 (1987), 325-334.

19. M. S. Moslehian, A. Zamani and M. Dehghani, Characterizations of smooth spaces by $\rho_{*-}$ orthogonality, Houston J. Math. 43 (2017), no. 4, 1187-1208.

20. G. J. Murphy, $C^{*}$-Algebras and Operator Theory, Academic Press, New York, 1990.

21. D. Werner, An elementary approach to the Daugavet equation, in: Interaction Between Functional Analysis, Harmonic Analysis, and Probability (Columbia, MO, 1994), Lecture Notes in Pure and Applied Mathematics, 175 (Marcel Dekker, New York, 1996), 449-454.

22. P. Wójcik, Linear mappings preserving $\rho$-orthogonality, J. Math. Anal. Appl. 386 (2012), 171-176.

23. P. Wójcik, Linear mappings approximately preserving orthogonality in real normed spaces, Banach J. Math. Anal. 9, no. 2, (2015), 134-141.

24. P. Wójcik, Gateaux derivative of the norm in $\mathcal{K}(X ; Y)$, Ann. Funct. Anal. 7 (2016), no. 4, $678-685$. 
25. P. Wójcik, Norm-parallelism in classical M-ideals, Indag. Math. (N.S.) 28(2) (2017), 287293.

26. P. Wójcik, Birkhoff orthogonality in classical M-ideals, J. Aust. Math. Soc. 103 (2017), 279-288.

27. P. Wójcik, Generalized Daugavet equations, affine operators and unique best approximation, Stud. Math. 238(3) (2017), 235-247.

28. P. Wójcik, Extensions of linear operators from hyperplanes and strong uniqueness of best approximation in $\mathcal{L}(X, W)$, J. Approx. Theory 246 (2019), 28-42.

29. P. Wójcik, Characterization of linear similarities through functional equation and mappings preserving orthogonalities, Linear Algebra Appl. 579 (2019), 206-216.

30. A. Zamani and M. S. Moslehian, Exact and approximate operator parallelism, Canad. Math. Bull. 58(1) (2015), 207-224.

31. A. Zamani and M. S. Moslehian, Norm-parallelism in the geometry of Hilbert $C^{*}$-modules, Indag. Math. (N.S.) 27(1) (2016), 266-281.

32. A. Zamani and M. S. Moslehian, An extension of orthogonality relations based on norm derivatives, Q. J. Math. 70 (2019), no. 2, 379-393.

${ }^{1}$ Institute of Mathematics, Pedagogical University of Cracow, PodchorA̧żych 2 , 30-084 Kraków, POLAND

Email address: pawel.wojcik@up.krakow.pl

* Corresponding author, ${ }^{2}$ School of Mathematics and Computer Sciences, DAmghan University, DAmghan, P. O. BOX 36715-364, IrAN

Email address: zamani.ali85@yahoo.com 